\title{
Label-free Amperometric Immunosensor for Quantitative Detection of Low-concentration Brucella-positive Standard Serum
}

\author{
Wei Yang, Zhiyong Zhang, Haiyan Song, Xiaoping Han, and Yueming Zuo* \\ College of Engineering, Shanxi Agricultural University, Taigu 030801, P.R. China
}

(Received November 9, 2018; accepted January 28, 2019)

Keywords: Brucella-positive standard serum, label-free amperometric immunosensor, quantitative detection, voltammogram, second-derivative transformation

Brucella bacteria are widely distributed throughout the world causing huge losses in agriculture and threatening human health through zoonotic infection. In this article, a label-free amperometric immunosensor for the quantitative detection of a low-concentration Brucellapositive standard serum was developed to identify diseased livestock earlier and thereby reduce economic losses. The developed immunosensor consists of a screen-printed gold electrode (SPGE) and a signal-acquiring interface. The SPGE was first functionalized with cysteamine for the immobilization of Brucella melitensis. Then, Brucella-positive standard sera of various concentrations were introduced dropwise onto the surface of the electrode and captured by $B$. melitensis. This resulted in current changes that could be measured with differential pulse and square wave voltammetries in the electrolyte solution using an electrochemical workstation. There was a linear relationship between the variation in peak current and the logarithmic value of Brucella-positive standard serum concentrations from $1 \times 10^{-5}$ to $1 \times 10^{2}$ $\mathrm{IU} / \mathrm{mL}$. Furthermore, the second-derivative transformation was applied, and the optimized linear correlation between the variation in peak value and the corresponding Brucella-positive standard serum concentration was established. Moreover, the regression equation has a lower detection limit, a higher goodness of fit, and a smaller residual sum of squares.

\section{Introduction}

Brucellosis is a worldwide zoonotic infectious disease caused by Brucella bacteria. ${ }^{(1,2)}$ Domestic animals often show effects such as miscarriage, stillbirth, and infertility. ${ }^{(3)}$ Brucellosis is often transmitted to humans through direct or indirect contact with infected animals or their products. ${ }^{(4)}$ People with a brucellosis often suffer from high fever, night sweats, arthritis, and endocarditis with long duration of illness, and may even become severely disabled or immobile. ${ }^{(5)}$ Brucellosis causes huge losses in agricultural production and serious public health problems. ${ }^{(6,7)}$ Currently, Brucellosis is typically diagnosed by bacteriological and serological methods. The bacteriological detection method is reliable and accurate, but it requires a long time. Therefore, the serological test method has an indisputable advantage.

*Corresponding author: e-mail: zuoyueming@163.com https://doi.org/10.18494/SAM.2019.2180 
Serological detection methods include the standard tube agglutination test (SAT), plate agglutination test (PAT), rose bengal plate test (RBPT), complement fixation test (CFT), and enzyme-linked immunosorbent assay (ELISA). ${ }^{(8-10)}$ However, these methods often have some drawbacks, such as a low sensitivity, a complex process, a long processing time, laborious techniques, and even potential biological risks. ${ }^{(11,12)}$ Moreover, most of these procedures have been adopted for qualitative or semiquantitative detection in Brucella assays. ${ }^{(13)}$ Additionally, not all infected animals have the level of significance as that of the diagnostic antibodies. Therefore, it is necessary to detect low Brucella-positive standard serum concentrations for the early diagnosis of brucellosis. In order to overcome the above shortcomings, many novel rapid methods are being developed to replace traditional techniques, by combining diverse capture methods, and also, many detection technologies are being developed to improve the total assay time and further amplify the signals. ${ }^{(14)}$ The use of an immunosensor is effective for detecting antibodies. ${ }^{(15)}$ For instance, the amperometric enzyme-linked immunosensor has been applied by using resveratrol as the substrate for horseradish peroxidase to detect the Brucella-positive standard serum. ${ }^{(16)}$ In particular, Nauman et al. reported that the second derivative method was successfully applied to the quantification of urea in urine, ${ }^{(17)}$ and that appropriate mathematical methods can effectively improve the quantification of target compounds.

In this study, we focused on improving the sensing of a low Brucella-positive standard serum concentration by these mathematical methods. A label-free amperometric immunosensor for the detection of the Brucella-positive standard serum was developed. It was based on the immobilization of Brucella melitensis on the surface of a screen-printed gold electrode (SPGE) modified by cysteamine (CA)/glutaraldehyde (GA). CA could form a self-assembled monolayer on the SPGE and bond with antigens, enabling the detection of different concentrations of antibodies by differential pulse voltammetry (DPV) and square wave voltammetry (SWV). The linear relationship between the peak current increment of the test curve and each concentration was established. The second-derivative transformation of the test data was simultaneously performed to optimize the linear relationship. This research is significant for realizing the detection of a low Brucella-positive standard serum concentration.

\section{Materials and Methods}

\subsection{Reagents}

$\mathrm{CA}$ and $25 \%$ GA were purchased from Sigma-Aldrich (Beijing, China). Bovine serum albumin (BSA) was purchased from Roche Diagnostics GmbH (Mannheim, Germany). $\mathrm{K}_{4} \mathrm{Fe}(\mathrm{CN})_{6}, \mathrm{~K}_{3} \mathrm{Fe}(\mathrm{CN})_{6}$, and $\mathrm{KCl}$ were purchased from Solarbio Science \& Technology Co., Ltd. (Beijing, China). B. melitensis $\left(4 \times 10^{10} \mathrm{CFU} / \mathrm{mL}\right)$ and a Brucella-positive standard serum $\left(1 \times 10^{3} \mathrm{IU} / \mathrm{mL}\right)$ were purchased from the China Institute of Veterinary Drug Control (Beijing, China). Other chemicals used were of analytical grade and used without further purification.

The following solutions were prepared: phosphate buffered saline (PBS, $10 \mathrm{mM}, \mathrm{pH}$ 7.4), blocking buffer (1\% BSA in PBS), GA solution (2.5\% GA in PBS), CA solution (10 mM CA in ethanol), electrolyte solution [2.5 $\mathrm{mM} \mathrm{K}{ }_{4} \mathrm{Fe}(\mathrm{CN})_{6}, 2.5 \mathrm{mM} \mathrm{K}_{3} \mathrm{Fe}(\mathrm{CN})_{6}$, and $0.1 \mathrm{M} \mathrm{KCl}$ in PBS], and sulfuric acid solution ( $0.5 \mathrm{M} \mathrm{98 \%} \mathrm{sulfuric} \mathrm{acid} \mathrm{in} \mathrm{deionized} \mathrm{water).}$ 


\subsection{Apparatus}

Voltammetry tests were performed on an electrochemical workstation provided by Shanghai Chenhua Instrument Inc. (model CHI660C, Shanghai, China). The pH of the solutions was measured with a pH meter obtained from Sartorius Instrument, Inc. (Model PB-10, Germany). SPGEs were purchased from DropSens, Inc. (model X250BT, Oviedo, Spain). The SPGEs consisted of a gold working electrode (WE), a platinum counter electrode (CE), and a silver reference electrode $(\mathrm{RE})$ on a ceramic substrate $\left[34(L) \times 10(W) \times 0.5(H) \mathrm{mm}^{3}\right]$. All solutions were prepared with deionized water in a millipore water purification system (Milli-Q Advantage A10, USA). All electrochemical experiments were performed at room temperature $\left(25 \pm 1^{\circ} \mathrm{C}\right)$.

\subsection{Electrode pretreatment}

To eliminate the potential oxidation of the electrode surface, $50 \mu \mathrm{L}$ of sulfuric acid was deposited evenly onto the electrode surface. The cyclic voltammetry (CV) experiment was scanned in the voltage range of -0.1 and $1.5 \mathrm{~V}$ at a rate of $100 \mathrm{mV} / \mathrm{s}$ with 8 cycles. Subsequently, the cleaned electrode was thoroughly rinsed with deionized water.

\subsection{Preparation of microbial sample}

The series diluents of B. melitensis were antigens, and the Brucella-positive standard sera were antibodies. The antigens and antibodies were dissolved with PBS before use. The concentration of the antigens was $4 \times 10^{9} \mathrm{CFU} / \mathrm{mL}$. The concentrations of the antibodies were $1 \times 10^{-5}, 1 \times 10^{-3}, 1 \times 10^{-1}, 1 \times 10^{1}$, and $1 \times 10^{2} \mathrm{IU} / \mathrm{mL}$. To avoid contamination, the dilutions of antigens and antibodies were prepared in an ultraclean working platform.

\subsection{Fabrication of immunosensors}

Each pretreated SPGE was immersed in CA for $120 \mathrm{~min}$ in a closed environment at room temperature; then, the electrode surface was cleaned with ethanol and deionized water twice to remove the unbound $\mathrm{CA} .^{(18)}$ Next, $10 \mu \mathrm{L}$ of $2.5 \%$ GA was coated on the WE surface for 60 min at $37{ }^{\circ} \mathrm{C}$ in a sterile and wet airtight container. Then, the combination was rinsed with PBS and deionized water and dried for $10 \mathrm{~min}$ at room temperature. Subsequently, $10 \mu \mathrm{L}$ of the antigen solution was pipetted directly onto the WE of the SPGE and incubated for $60 \mathrm{~min}$ at $37{ }^{\circ} \mathrm{C}$ in a sterile and wet airtight container, followed by cleaning using PBS and drying as in the previous steps. Finally, $10 \mu \mathrm{L}$ of BSA was pipetted directly onto the WE of the SPGE and incubated for $60 \mathrm{~min}$ at $37{ }^{\circ} \mathrm{C}$ in a sterile and wet airtight container to block nonspecific binding sites, followed by rinsing with deionized water to remove the unbounded BSA. Hence, the antigens were immobilized on the modified electrode, and the immunosensors were fabricated. 


\subsection{Measurement procedure}

The fabricated SPGEs were characterized by CV to establish the stability of the current intensity. The CV tests were performed between -0.3 and $0.6 \mathrm{~V}$ at $100 \mathrm{mV} / \mathrm{s}$.

The antibody concentrations were determined from the variation in the peak current of the immunosensors $\left[I_{p a(B S A)}-I_{p a(A n t i b o d y)}\right]$ by DPV and SWV. The sweep potential of DPV between -0.15 and $0.35 \mathrm{~V}$ was applied with a voltage increment of $0.004 \mathrm{~V}$ and an amplitude of $0.05 \mathrm{~V}$ at a pulse period of $0.2 \mathrm{~s}$. In the SWV experiment, scanning was from -0.15 to $0.45 \mathrm{~V}$, the pulse amplitude was set at $0.025 \mathrm{~V}$, the potential increment was $0.004 \mathrm{~V}$, and the frequency of SWV was $15 \mathrm{~Hz}$. All the current and potential data were recorded on an electrochemical station with $50 \mu \mathrm{L}$ of the supporting electrolyte applied to the electrochemical reaction area.

The immunosensors were coated with different antibody concentrations of $1 \times 10^{-5}$, $1 \times 10^{-3}, 1 \times 10^{-1}, 1 \times 10$, and $1 \times 10^{2} \mathrm{IU} / \mathrm{mL}$ and then incubated for $30 \mathrm{~min}$ at $37^{\circ} \mathrm{C}$. The immunosensors were tested by DPV and SWV with the above procedure.

For further improvement, we transformed the test curve by the second-derivative transformation. The derivative transformation was accomplished using ${ }^{(19)}$

$$
\frac{d y}{d x}(n)=0.5\left(\frac{y(n)-y(n-1)}{x(n)-x(n-1)}\right)+0.5\left(\frac{y(n-1)-y(n)}{x(n-1)-x(n)}\right),
$$

where $y$ is the current, $x$ is the potential, and $n$ is each of the data points. We used the secondderivative transformation signal as it was more efficient in eliminating the background interferences. ${ }^{(20)}$

\subsection{Data analysis}

Each determination of antibodies was repeated seven times using different SPGEs to test the reproducibility of the immunosensor. When different concentrations of antibodies were tested, the peak current would change after an immune reaction between immobilized antigens and tested antibodies. All data were presented as the mean and standard deviation values. All coefficients of determination were analyzed by the t-test, and the differences between groups were evaluated using the $P$-value. The standard deviation was calculated, the blank solution test was repeated 3 times, and the response value of the threefold standard deviation was substituted into the regression curve to obtain the minimum detection limit. ${ }^{(21)}$ The data were analyzed with the Origin Lab 8.5 software.

\section{Results and Discussion}

\subsection{Optimization of process parameters}

The antigen concentrations of $4 \times 10^{8}$ and $4 \times 10^{9} \mathrm{CFU} / \mathrm{mL}$ were selected to bind to the modified electrode. The oxidation peak currents of binding the $4 \times 10^{8}$ and $4 \times 10^{9} \mathrm{CFU} / \mathrm{mL}$ 
antigens decreased by 6.1 and $9.1 \mu \mathrm{A}$, respectively. This indicated that more antigens were linked to the interface after binding with $4 \times 10^{9} \mathrm{CFU} / \mathrm{mL}$ antigens, which could provide more binding sites for the immune reaction of antibodies. Therefore, $4 \times 10^{9} \mathrm{CFU} / \mathrm{mL}$ was selected as the binding concentration of antigens.

$\mathrm{pH}$ strongly affected the antigen-antibody affinity. Most of the antigen-antibody responses were suitable for the $\mathrm{pH}$ range of 6 to 8 . In this experiment, we selected a $\mathrm{pH}$ of 7.4, which is similar to the $\mathrm{pH}$ of the living environment of the pathogen in organisms; it was more suitable for immune reactions.

The immune response had a certain dependence on temperature. Because immune components are generally active at animal body temperatures and occasionally show increased sensitivity, ${ }^{(22)} 37^{\circ} \mathrm{C}$ was selected as the incubation temperature of the immune reaction.

\subsection{Electrochemical characteristics of the electrode}

The potential value between oxidation and reduction peaks $\left(\Delta E_{p}\right)$, the absolute value of the ratio of oxidation peak current to reduction peak current $\left(\left|I_{p a} / I_{p c}\right|\right)$, and the oxidation peak current $\left(I_{p a}\right)$ were the characteristic parameters of the electrode. The electrode was tested by $\mathrm{CV}$ in the supporting electrolyte, and the electrode pretreatment and modification processes were analyzed in reference to the changes in the characteristic parameters. The electrochemical properties of the electrode were considerably improved by the pretreatment and modification of the bare electrode (Fig. 1). As shown in Table 1, compared with the bare electrode, the $I_{p a}$ of the pretreated electrode increased from 69.15 to $118.30 \mu \mathrm{A}, \Delta E_{p}$ decreased from 217.67 to $89.00 \mathrm{mV}$, and the relative standard deviation (RSD) of $\left|I_{p a} / I_{p c}\right|$ decreased from 1.2 to 0.5 . Compared with the pretreated electrode, the $I_{p a}$ of the modified electrode increased from 118.30 to $132.80 \mu \mathrm{A}$, $\Delta E_{p}$ decreased from 89.00 to $62.33 \mathrm{mV}$, and the RSD of $\left|I_{p a} / I_{p c}\right|$ decreased to 0.2 . These results showed that the electrochemical characteristics of the pretreated and modified electrodes were better than those of the bare electrode, and the conductivity of the SPGE was enhanced. The increase in oxidation peak current was beneficial in improving the sensitivity and repeatability

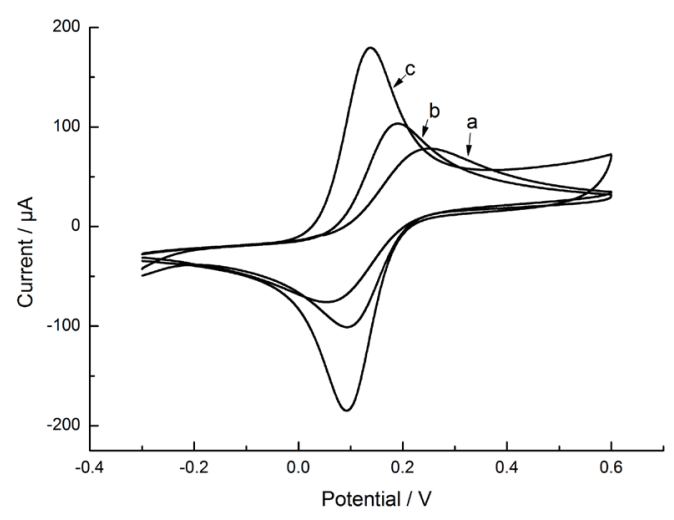

Fig. 1. CVs of the SPGEs obtained in electrolyte solution under the following conditions: (a) bare SPGE, (b) after SPGE was pretreated, and (c) after modifying SPGE with CA. 
Table 1

Characteristic parameters of the electrode.

\begin{tabular}{lccc}
\hline & Electrode & Measured value & RSD $(\%)$ \\
\hline \multirow{4}{*}{$I_{p a}(\mu \mathrm{A})$} & Bare electrode & $69.15 \pm 2.32$ & 3.3 \\
& Pretreated electrode & $118.30 \pm 3.34$ & 2.8 \\
& Electrode modified with CA & $132.80 \pm 4.65$ & 3.5 \\
& Bare electrode & $217.67 \pm 7.57$ & 3.4 \\
\hline \multirow{2}{*}{$\Delta E_{p}(\mathrm{mV})$} & Pretreated electrode & $89.00 \pm 4.36$ & 4.8 \\
& Electrode modified with CA & $62.33 \pm 4.16$ & 6.6 \\
& Bare electrode & $1.01 \pm 0.01$ & $1.2^{1}$ \\
\hline \multirow{2}{*}{$\left|I_{p a} / I_{p c}\right|$} & Pretreated electrode & $0.98 \pm 0.01$ & 0.5 \\
& Electrode modified with CA & $1.01 \pm 0.01$ & $0.2^{1}$ \\
\hline
\end{tabular}

${ }^{1}$ To retain a unified decimal number, the data were rounded off, which results in the same measured value, but different RSDs.

of the immunosensor, which had sufficient current to meet the requirements of further tests. This indicated that the presence of the modified electrode promoted electron transfer and enhanced the conductivity.

\subsection{Detection of antigen-antibody interaction by voltammograms}

The antigen-antibody immune reaction was tested by DPV and SWV. Figure 2 shows that the peak current decreased with increasing antibody concentration. On the electrode surface, a nonconductive layer that blocked the channel of electron transport and thus hindered the transmission of the electrolyte was formed. ${ }^{(23)}$

Five concentrations of antibodies were tested. The linear relationship between the logarithmic value of antibody concentrations and $\Delta I_{p}$ is shown in Fig. 3. The fitted curve of SWV showed a lower detection limit of immunosensors than that of DPV. In SWV, because the electrolytic current is recorded only when the charging current is sufficiently attenuated, the influence of background current can be effectively eliminated. ${ }^{(24)}$ Therefore, the fitted curve of the SWV resolution is higher. SWV is a sensitive technique for measuring the current by sensing the changes on the electrode surface.

The regression equation, the residual sum of squares (RSS), the limit of detection (LOD), and the coefficient of determination $\left(R^{2}\right)$ are shown in Table 2 . When the antibody concentrations range from $1 \times 10^{-5}$ to $1 \times 10^{2} \mathrm{IU} / \mathrm{mL}$, the fitted curves were well distinguished among the five different concentrations of antibody solutions. The minimum detection limits were $7.3922 \times 10^{-6}$ and $3.7601 \times 10^{-6} \mathrm{IU} / \mathrm{mL}$ for DPV and SWV, respectively.

\subsection{Second-derivative transformation of voltammograms}

The improvements in the detection limit and coefficient of determination were critical for the immunosensors. We transformed the test curve by second-derivative transformation, as 


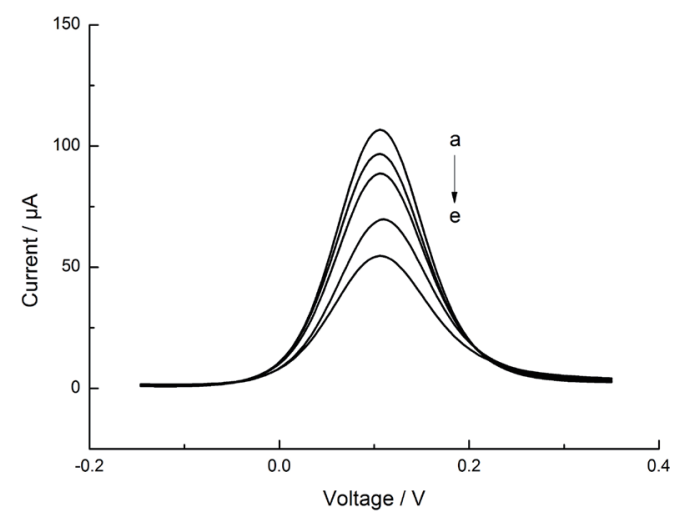

(a)

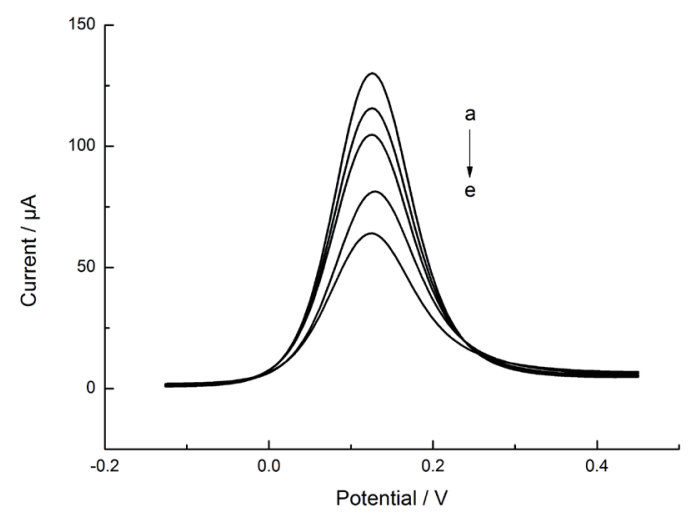

(b)

Fig. 2. (a) DPV and (b) SWV results for immunosensor. Concentrations of antibodies: a, $1 \times 10^{-5}$; b, $1 \times 10^{-3}$; c, $1 \times 10^{-1} ; \mathrm{d}, 1 \times 10^{1}$; and e, $1 \times 10^{2} \mathrm{IU} / \mathrm{mL}$.

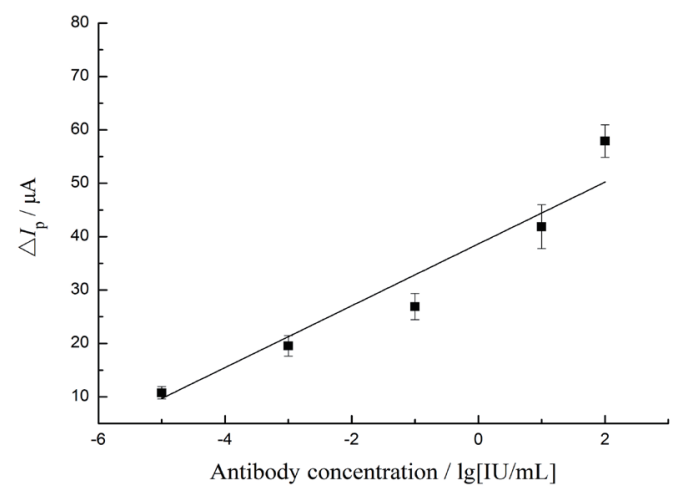

(a)

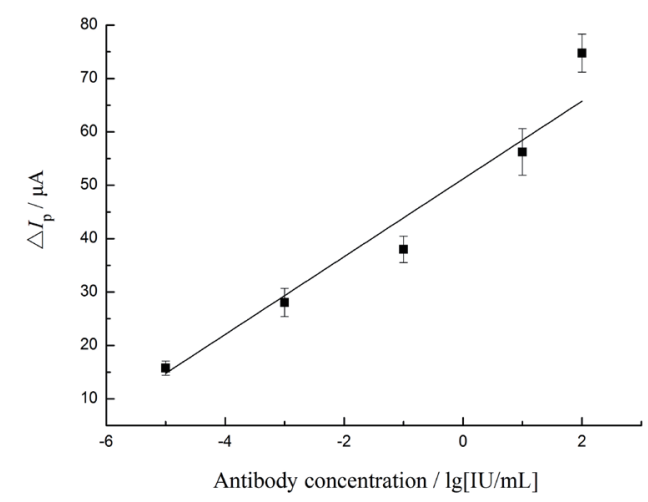

(b)

Fig. 3. Fitted curves for (a) DPV and (b) SWV, which show the range of concentrations between $1 \times 10^{-5}$ and $1 \times$ $10^{2} \mathrm{IU} / \mathrm{mL}$. The means and standard error bars are shown.

Table 2

Related parameters of fitting curve for different antibody concentrations.

\begin{tabular}{lcccccc}
\hline $\begin{array}{l}\text { Test } \\
\text { method }\end{array}$ & $\begin{array}{c}\text { Concentration } \\
\text { range (IU/mL) }\end{array}$ & Regression equation & RSS & LOD $(\mathrm{IU} / \mathrm{mL})$ & $R^{2}$ & $P$-value \\
\hline DPV & $10^{-5}-10^{2}$ & $Y=38.6459+5.7886 \lg C$ & 14.4044 & $7.3922 \times 10^{-6}$ & 0.92 & $<0.01$ \\
SWV & $10^{-5}-10^{2}$ & $Y=51.1831+7.2803 \lg C$ & 13.2021 & $3.7601 \times 10^{-6}$ & 0.94 & $<0.01$ \\
\hline
\end{tabular}

shown in Fig. 4. Figure 5 shows the linear relationship between the increment of the single peak and the various antibody concentrations. As shown in Table 3, when the antibody concentration was between $1 \times 10^{-5}$ and $1 \times 10^{2} \mathrm{IU} / \mathrm{mL}$, the fitted curves were well distinguished between the five different concentrations of antibody solutions. The minimum detection limits were $1.1182 \times 10^{-8}$ and $1.3041 \times 10^{-8} \mathrm{IU} / \mathrm{mL}$ for DPV and SWV, respectively. The second-derivative transformation of voltammograms could maintain better peak-shaped signals and amplify the current signal compared with that of only the original voltammograms. The data showed that 


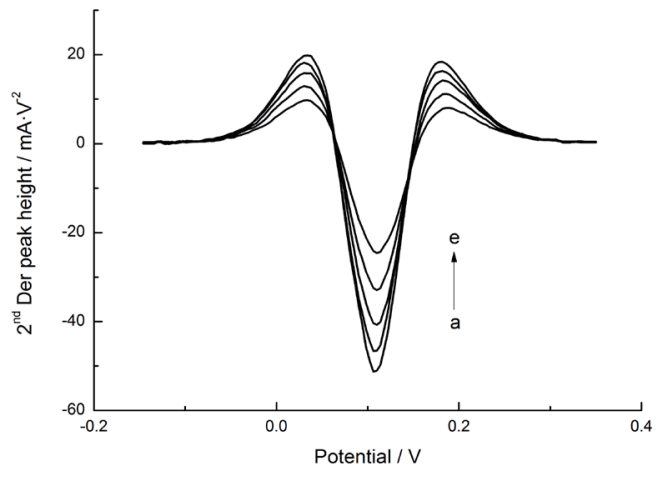

(a)

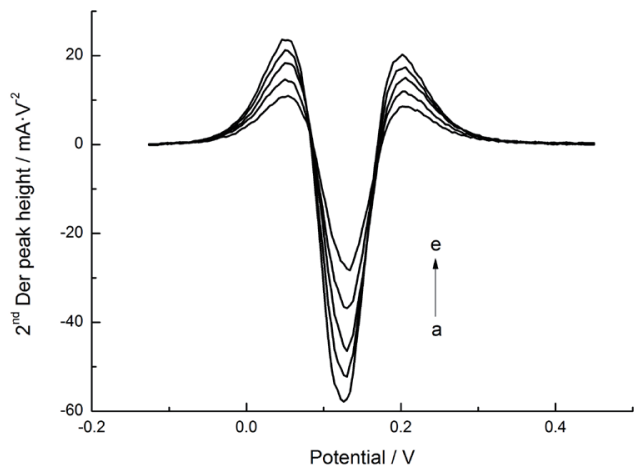

(b)

Fig. 4. (a) DPV and (b) SWV second-derivative transformation curves. Concentrations of antibodies: a, $1 \times 10^{-5} ; \mathrm{b}$, $1 \times 10^{-3} ; \mathrm{c}, 1 \times 10^{-1} ; \mathrm{d}, 1 \times 10^{1}$; and $\mathrm{e}, 1 \times 10^{2} \mathrm{IU} / \mathrm{mL}$.

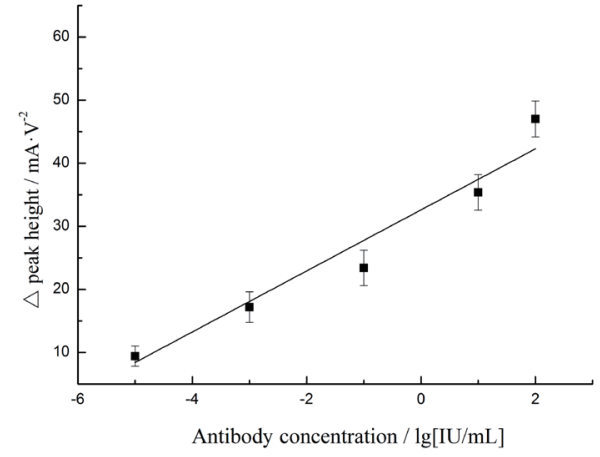

(a)

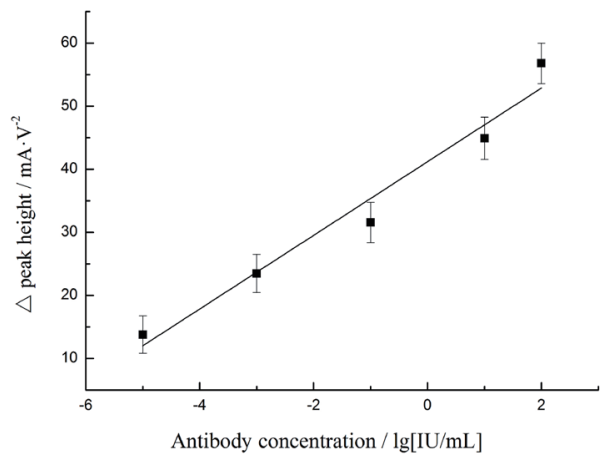

(b)

Fig. 5. Fitted curves of (a) DPV and (b) SWV second-derivative transformations. The means and standard error bars are shown. The fitted curves of (a) and (b) show the range of concentrations between $1 \times 10^{-5}$ and $1 \times 10^{2}$ $\mathrm{IU} / \mathrm{mL}$.

Table 3

Related parameters of fitted curve for different antibody concentration ranges after second-derivative transformation.

\begin{tabular}{lcccccc}
\hline $\begin{array}{l}\text { Second-derivative } \\
\text { transformation } \\
\text { of voltammogram }\end{array}$ & $\begin{array}{c}\text { Concentration } \\
\text { range }(\mathrm{IU} / \mathrm{mL})\end{array}$ & Regression equation & RSS & LOD (IU/mL) & $R^{2}$ & $P$-value \\
\hline DPV & $10^{-5}-10^{2}$ & $Y=32.6139+4.8363 \lg C$ & 6.2179 & $1.1182 \times 10^{-8}$ & 0.94 & $<0.01$ \\
SWV & $10^{-5}-10^{2}$ & $Y=41.2064+5.8370 \lg C$ & 3.6522 & $1.3041 \times 10^{-8}$ & 0.95 & $<0.01$ \\
\hline
\end{tabular}

the second-derivative transformation of voltammograms had lower detection limits, a higher goodness of fit, and a smaller RSS. The second-derivative transformation was found to be sensitive and accurate. ${ }^{(17)}$ The proposed method was successfully applied in the quantitative assay of antibodies. 


\section{Conclusions}

We developed an immunosensor for the quantification of a Brucella-positive standard serum. On SPGEs, a self-assembled monolayer of CA was formed, and the bonded antigens could be used to test different concentrations of antibodies by DPV and SWV. The linear relationship between the peak current increment of the test curve and the concentration was established. For concentrations between $1 \times 10^{-5}$ and $1 \times 10^{2} \mathrm{IU} / \mathrm{mL}$, the minimum detection limits were $7.3922 \times 10^{-6}$ and $3.7601 \times 10^{-6} \mathrm{IU} / \mathrm{mL}$, respectively. The second-derivative transformation of the test data was simultaneously performed to establish the linear relationship between the increment of the single peak and the concentration of each antibody. The minimum detection limits were $1.1182 \times 10^{-8}$ and $1.3041 \times 10^{-8} \mathrm{IU} / \mathrm{mL}$ for DPV and SWV, respectively. The corresponding regression equation had a lower LOD, a higher goodness of fit, and a smaller RSS. The results sufficiently showed that appropriate mathematical methods could effectively improve sensing results. This study could provide a reference for the detection of lowconcentration target antibodies. However, how to use this method in reality must still be studied further.

\section{Acknowledgments}

This work was supported by the National Natural Science Foundation of China through grant number 30871445, the Shanxi Scholarship Council of China through grant number 2013061, and the Shanxi Agricultural University Youth Science and Technology Innovation Fund Project of China through grant number 201503.

\section{References}

1 H. H. Al-Griw, E. S. Kraim, M. E. Farhat, L. L. Perrett, and A. M. Whatmore: J. Epidemiol. Global Health. 7 (2017) 285. https://doi.org/10.1016/j.jegh.2017.09.001

2 M. O. Ahmed, Y. M. Abouzeed, E. M. Bennour, and J. C. van Velkinburgh: Pathog. Global Health. 109 (2015) 39. https://doi.org/10.1179/2047773214Y.0000000170

3 W. Beauvais, R. J. Coker, G. Nurtazina, and J. Guitian: EcoHealth 14 (2017) 399. https://doi.org/10.1007/ s10393-015-1030-7

4 M. Ducrotoy, W. J. Bertu, G. Matope, S. Cadmus, R. Conde-Álvarez, A. M. Gusi, S. Welburn, R. Ocholi, J. M. Blasco, and I. Moriyón: Acta Trop. 165 (2017) 179. https://doi.org/10.1016/j.actatropica.2015.10.023

5 T. Buzgan, M. K. Karahocagil, H. Irmak, A. I. Baran, H. Karsen, O. Evirgen, and H. Akdeniz: Int. J. Infect. Dis. 14 (2010) e469. https://doi.org/10.1016/j.ijid.2009.06.031

6 J. A. Assenga, L. E. Matemba, S. K. Muller, J. J. Malakalinga, and R. R. Kazwala: BMC Vet. Res. 11 (2015) 189. https://doi.org/10.1186/s12917-015-0504-8

7 C. M. Dentinger, K. Jacob, L. V. Lee, H. A. Mendez, K. Chotikanatis, P. L. McDonough, D. M. Chico, B. K. De, R. M. Traxler, E. R. Campagnolo, D. Schmitt, M. A. Guerra, and S. A. Slavinski: Zoonoses Public Health. 62 (2015) 407. https://doi.org/10.1111/zph.12163

8 A. Praud, M. Durán-Ferrer, D. Fretin, M. Jaÿ, M. O'Connor, A. Stournara, M. Tittarelli, I. T. Dias, and B. Garin-Bastuji: Vet. J. 216 (2016) 38. https://doi.org/10.1016/j.tvj1.2016.06.014

9 S. O'Leary, M. Sheahan, and T. Sweeney: Res. Vet. Sci. 81 (2006) 170. https://doi.org/10.1016/j.rvsc.2005.12.001

10 S. Mitka, C. Anetakis, E. Souliou, E. Diza, and A. Kansouzidou: J. Clin. Microbiol. 45 (2007) 1211. https://doi. org/10.1128/JCM.00010-06

11 H. Hanci, H. Igan, and M. H. Uyanik: Pak. J. Biol. Sci. 20 (2017) 108. https://doi.org/ 10.3923/pjbs.2017.108.112

12 L. Li, D. H. Yin, K. Xu, Y. S. Liu, D. D. Song, J. Wang, C. Zhao, X. L. Song, and J. Li: J. Pharm. Biomed. Anal. 141 (2017) 79. https://doi.org/10.1016/j.jpba.2017.03.002

13 K. Szulowski, W. Iwaniak, J. Pilaszek, M. Truszczyński, and M. Chrobocińska: Comp. Immunol. Microbiol. 
Infect. Dis. 22 (1999) 33. https://doi.org/10.1016/S0147-9571(98)00019-8

14 Y. C. Liu and Y. B. Li: J. Microbiol. Methods 51 (2002) 369. https://doi.org/10.1002/ppul.21027

15 W. Yang, Y. M. Zuo, H. Y. Wu, Z. Y. Zhang, R. Zhang, and C. Chen: Trans. Chin. Soc. Agric. Mach. 45 (2014) 243. https://doi.org/10.6041/j.issn.1000-1298.2014.03.040

16 F. C. Gong, D. S. He, Z. Cao, S. Z. Tan, and Y. F. Tan: Chin. J. Anal. Chem. 35 (2007) 1783. https://doi. org/10.1016/s1872-2040(08)60009-2

17 N. Ali, M. Ismail, A. Khan, H. Khan, S. Haider, and T. Kamal: Spectrochim. Acta, Part A 189 (2018) 110. https://doi.org/10.1016/j.saa.2017.07.063

18 B. B. Luo, Y. F. Xu, S. X. Wu, M. F. Zhao, P. J. Jiang, S. H. Shi, Z. H. Zhang, Y. Wang, L. L. Wang, and Y. Liu: Biosens. Bioelectron. 100 (2018) 169. https://doi.org/ 10.1016/j.bios.2017.08.064

19 A. Cobelo-García, J. Santos-Echeandía, D. E. López-Sánchez, C. Almécija, and D. Omanović: Anal. Chem. 86 (2014) 2308. https://doi.org/10.1021/ac403558y

20 C. E. Monteiro, A. Cobelo-Garcia, M. Caetano, and M. M. Correia Dos Santos: Talanta 175 (2017) 1. https:// doi.org/10.1016/j.talanta.2017.06.067

21 H. Y. Wu, Y. M. Zuo, C. J. Cui, W. Yang, H. L. Ma, and X. W. Wang: Sensors 13 (2013) 8551. https://doi. org/10.3390/s130708551

22 M. Ashaduzzaman, A. A. Antony, N. A. Murugan, S. R. Deshpande, A. P. Turner, and A. Tiwari: Biosens. Bioelectron. 73 (2015) 100. https://doi.org/10.1016/j.bios.2015.05.055

23 T. Wen, R. Wang, A. Sotero, and Y. Li: Sensors 17 (2017) 1973. https://doi.org/10.3390/s17091973

24 M. Hasanzadeh, H. N. Baghban, N. Shadjou, and A. Mokhtarzadeh: Int. J. Biol. Macromol. 107 (2018) 1348. https://doi.org/10.1016/j.ijbiomac.2017.11.006

\section{About the Authors}

Wei Yang received her B.S. degree from Shenyang Agricultural University, China, in 2004, and her M.S. and Ph.D. degrees from Shanxi Agricultural University, China, in 2007 and 2014, respectively. Since 2014, she has been a lecturer at Shanxi Agricultural University. Her research interests are in intelligent detection and sensor technology. (yw@sxau.edu.cn)

Zhiyong Zhang received his B.S. and M.S. degrees from Shanxi Agricultural University, China, in 2001 and 2005, respectively, and his Ph.D. degree from Tianjin University, China, in 2012. Since 2013, he has been an associate professor at Shanxi Agricultural University, China. His research interests are in the rapid detection of agricultural product quality and automatic control and detection of agricultural equipment.

Haiyan Song received her B.S. and M.S. degrees from Shanxi Agricultural University, China, in 2000 and 2003, respectively, and her Ph.D. degree from Zhejiang University, China, in 2006. Since 2014, she has been a professor at Shanxi Agricultural University, China. Her research interests are in bio-environment monitoring technology and equipment, and automatic control and detection of agricultural equipment.

Xiaoping Han received her B.S. and M.S. degrees from Shanxi Agricultural University, China, in 2000 and 2004, respectively, and her Ph.D. degree from Shanxi Agricultural University, China, in 2011. Since 2013, she has been an associate professor at Shanxi Agricultural University, China. Her research interests are in agricultural bio-environment engineering and non-destructive testing technology. 
Yueming Zuo received his B.S. and M.S. degrees from Shanxi Agricultural University, China, in 1978 and 1981, respectively. Since 2001, he has been a professor at Shanxi Agricultural University. His research interests are in intelligent detection and sensor technology. (yuemingzuo@sxau.edu.cn) 